\title{
SPIN DYNAMICS IN AGS AND RHIC*
}

\author{
W. W. MacKay ${ }^{\dagger}$, L. Ahrens, M. Bai, K. Brown, E. D. Courant, J. W. Glenn, H. Huang, \\ A. U. Luccio, V. Ptitsyn, T. Roser,T. Satogata, S. Tepikian, N. Tsoupas, and A. N. Zelenski \\ BNL, Upton, NY 11973, USA.
}

\section{Abstract}

A fundamental aspect of particle physics is the spin of the particles. With polarized beams, the internal structure of the proton may be probed in ways that are unattainable with unpolarized beams. The Relativistic Heavy Ion Collider (RHIC) has the unique capability of colliding protons with both transverse and longitudinal polarization at centerof-mass energies up to $500 \mathrm{GeV}$. In this paper we examine the methods used to accelerate and manipulate polarized proton beams in RHIC and its injectors. Special techniques include the use of a partial Siberian snake and an ac dipole in the AGS. In RHIC we use four superconducting helical Siberian snakes (two per ring) for acceleration, and eight superconducting helical rotators for independent control of polarization directions at two interaction regions.

\section{INTRODUCTION}

The RHIC collider has been described in detail elsewhere[1], but it is worth noting (see Fig.1) the particular components used for spin control.

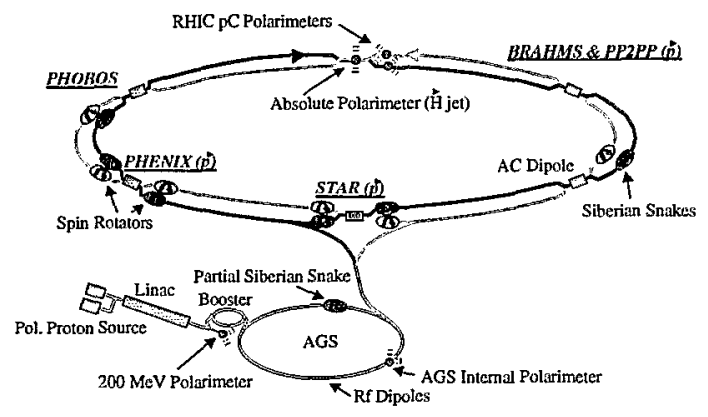

Figure 1: The accelerator complex for polarized protons. The beam in the RHIC Blue ring goes clockwise and in the Yellow ring counterclockwise.

An $\mathrm{H}^{-}$beam with polarized protons is produced by the optically pumped polarized ion source (OPPIS). The $\mathrm{H}^{-}$ beam is accelerated in the RFQ and linac to $200 \mathrm{MeV}$ $(\gamma=1.21)$. The beam is then stripped on injection to the Booster and accelerated to $2.46 \mathrm{GeV}(\gamma=2.62)$. Acceleration continues in the AGS up to $24.3 \mathrm{GeV}(\gamma=25.94)$ followed by transfer into either of the RHIC rings. There are several polarimeters to measure the polarization at various stages: one at the source, another at the end of the linac, a $\mathrm{p}+\mathrm{C} \mathrm{CNI}$ (Coulomb Nuclear Interference) polarimeters

\footnotetext{
*Work performed under the auspices of the U. S. Department of Energy (contract \# DE-ACO2-98CH10886) and RIKEN of Japan.

† waldo@bnl.gov
}

in the AGS and and each RHIC ring, as well as local polarimeters at the STAR and PHENIX detectors. There is a partial Siberian snake in the AGS and a pair of full Siberian snakes in each of the RHIC ring. Around each of the STAR and PHENIX detectors are four rotators as shown to manipulate the polarization direction through the experiment. There is an If dipole magnet in the AGS to aid in spin resonance crossing. RHIC has an additional ac dipole which will be used for spin flipping. A hydrogen jet polarimeter is presently under construction and will provide in the future an absolute calibration of the CNI polarimeters in RHIC.

\section{SPIN PRECESSION AND DYNAMICS}

In the local rest frame of the proton, the spin precession of the proton obeys the Thomas-Frenkel equation:[2]

$$
\begin{aligned}
\frac{d \vec{S}^{\circ}}{d t}=\frac{q}{\gamma m} \vec{S}^{\circ} \times & {\left[(1+G \gamma) \vec{B}_{\perp}+(1+G) \vec{B}_{\|}\right.} \\
& \left.+\left(G \gamma+\frac{\gamma}{\gamma+1}\right) \frac{\vec{E} \times \vec{v}}{c^{2}}\right] .
\end{aligned}
$$

This is a mixed description with $t, \vec{B}$, and $\vec{E}$ in the lab frame, but having the spin $\vec{S}^{\diamond}$ in local rest frame of the proton where the spatial components of $\vec{S}^{\circ}$ do not suffer the Lorentz lengthening of $S_{\|}$when boosted to the lab frame. Here the anomalous $g$-factor of the magnetic moment for the proton is

$$
G=\frac{g-2}{2}=1.7928
$$

If we consider the simple case of an idealized particle with a flat closed orbit and spin-component in the horizontal plane and only vertical magnetic fields, Eq. 1 simplifies to

$$
\begin{aligned}
\frac{d \vec{S}^{\diamond}}{d t} & =\frac{q}{\gamma m} \vec{S}^{\diamond} \times\left[(1+G \gamma) \vec{B}_{\perp}\right] . \\
\frac{d \vec{p}}{d t} & =\frac{q}{\gamma m} \vec{p} \times \vec{B}_{\perp} .
\end{aligned}
$$

is the Lorentz force for comparison. In this case the spin component precesses a factor of $1+G \gamma$ times faster than particle's velocity. A spin tune $\nu_{\mathrm{s}}$ can be defined the number of precessions of the spin in the particle's rest frame per turn (see Fig. 2a). In this case $\nu_{\mathrm{s}}=G \gamma$. It should be noted that for a simple ring $\nu_{\mathrm{s}}$ is proportional to energy.

A radial field component causing a vertical deflection of the particle by an angle $\alpha$ will cause the spin to precess about the radial direction by an angle $(1+G \gamma) \alpha$. Since rotations about different axes do not commute, such vertical excursions can modify the amount of precession and the 

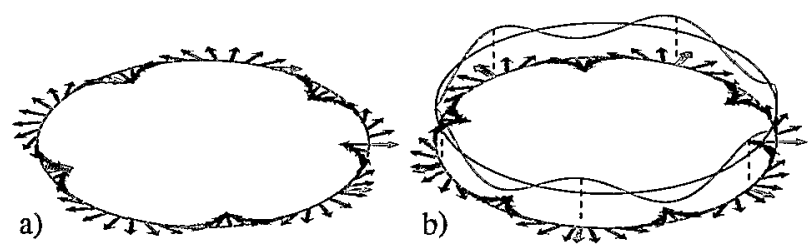

Figure 2: a) Conceptual plot of spin precession of the horizontal spin component for a spin tune of $\nu_{\mathrm{s}}=4.5$. b) Conceptual example of the intrinsic resonance $Q_{\mathrm{v}}=\nu_{\mathrm{s}}=5.5$.

spin tune. In addition the local axis about which the spins precess may move away from the vertical.

Integer (imperfection) resonances which are caused by misalignments of magnets can be quite strong and may flip the direction of polarization as the energy is ramped. For protons the energy separation of the imperfection resonances is $523 \mathrm{MeV}$.

The deflections of the vertical betatron oscillation also cause vertical precessions which can build up when the spin tune matches the vertical betatron tune $Q_{\mathrm{v}}$. (See Fig. 2b.) This type of resonance is called an intrinsic resonance. Clearly there can be coupling resonances where the horizontal tune comes into play, and in electron rings with large synchrotron oscillations, there are even synchrotron sidebands. For the most common resonances (intrinsic and imperfection) the resonance condition may be written as

$$
\nu_{\mathrm{s}}=N+N_{\mathrm{v}} Q_{\mathrm{v}},
$$

where $N$ and $N_{\mathrm{v}}$ are integers.

\section{Invariant spin field}

For the usual six dimensional phase space we have a closed orbit with the periodicity condition $\vec{q}_{0}(s+L)=$ $\vec{q}_{0}(s)$ where $L$ is the circumference of the ring, and the subscript zero indicates the closed-orbit trajectory. For the closed orbit, we can calculate a stable spin direction $\hat{n}_{0}(s)=\hat{n}_{0}(s+L)$. Other particles which are not on the closed orbit see different fields on every turn so that the amount of spin precession and direction will vary from turn to turn. For reasonable conditions we can still find a periodic condition for different locations in phase space, if we treat stable spin direction $\hat{n}(\vec{q}, \vec{p}, s)$ as a field associated with a point in phase space rather than being attached to individual particles.[3] For a given ensemble of particles the maximum possible polarization can not be more than the ensemble average of the spin field, although it may be less than this value. The individual particles may have spins which are not pointing in the direction of the corresponding $\hat{n}$-vector. Away from spin resonances the spin field vectors will be pointing in roughly the same direction, but near a resonance they can deviate quite wildly. Even though the polarization average of the ensemble may drop to zero near a resonance, the average may recover a nonzero value later when moving away from the resonance. In contrast to pro-

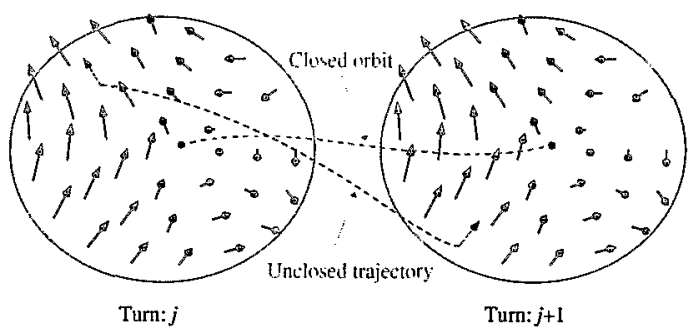

Figure 3: Illustration of the invariant spin field. For the closed orbit $\vec{n}_{0}(s)=\vec{n}_{0}(s+L)$, with coordinates $\vec{q}_{0}(s)=\vec{q}_{0}(s+L)$ and polarization direction $\vec{P}_{0}(s)=$ $\vec{P}_{0}(s+L)$. For other locations in phase space: $\vec{n}(\vec{q}, \vec{p}, s)=$ $\vec{n}(\vec{q}, \vec{p}, s+L)$, even though in general $q(s+L) \neq q(s)$ and $\vec{P}(s+L) \neq \vec{P}(s)$.

tons, electrons will lose this memory of polarization very quickly due to radiation effects.

\section{THE REAL MACHINES}

The linac provides a beam with about $70 \%$ polarization. In the Booster ( $G \gamma$ from 2.18 to 4.7 ), we cross two imperfection resonances by actually increasing the vertical closed orbit distortion to cause two total spin flips. We extract just below the first intrinsic resonance $\nu_{\mathrm{s}}=Q_{\mathrm{v}}=4.9$.

In the AGS with a more complicated resonance structure; the acceleration goes from $G \gamma=4.7$ up to 46.5 . Here we use a partial solenoidal snake to cause a larger lattice disturbance with a rotation of the spin about the longitudinal axis (see Eq. 1) and enhance spin flipping at the imperfection resonances. A single partial snake in the ring will open up stop bands in the spin tune at the integer as shown in Fig. 4a. Since a partial snake will tilt the stable spin direction away from the vertical, in the AGS we inject with the snake essentially off and then ramp it up to about $5 \%$ ( $9^{\circ}$ of rotation) for the ramp. At flattop, the snake is ramped down to about $0.3 \%$ (essentially off) for extraction to RHIC. With a single full snake in a ring the fractional part of $\nu_{\mathrm{s}}$ will be $\frac{1}{2}$, and we could expect the imperfection resonances go away. With a single full snake, the stable spin direction is in the horizontal plane which makes it difficult to match polarization from one ring to the next. As we increase energy, more precessions happen in the space between snake crossings, and the deleterious effects still build up, so that at RHIC energies up to $G \gamma=478(250 \mathrm{GeV})$ a single snake would not be enough to maintain a reasonable amount of polarization. Higher energies require more snakes.

In RHIC we typically inject and ramp with two full snakes on opposite sides of the rings (see Fig. 4b). Here the stable spin direction is vertical outside the snakes; a spin-up bunch in one half of the flips over at a snake to point down in the other half of the ring and flips back to up in the second snake.

The Froissart-Stora formula[4] gives the ratio of final to 

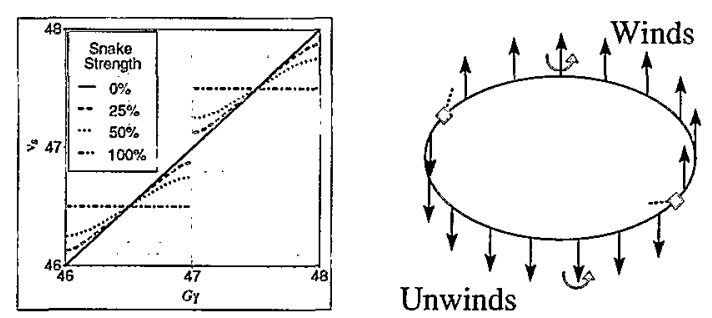

Figure 4: a) A single partial snake in a ring opens up stop bands for the spin tune. The percentage refers to the amount of spin rotation from the snake: $100 \%$ corresponds to $180^{\circ}$ of rotation. b) With two full snakes on opposite sides of the ring that have rotation axes perpendicular to each other the stable direction spin will be vertically up in one half of the ring and down in the other half, and the spin tune will be 0.5 .

initial polarization for an isolated resonance crossing:

$$
\frac{P_{\mathrm{f}}}{P_{\mathrm{i}}}=2 \exp \left(-\frac{\pi|\epsilon|^{2}}{2 \alpha}\right)-1,
$$

where $\alpha=d G \gamma / d \theta$ is the ramp rate in units of $G \gamma$ per radian of bend around the ring, and $\epsilon$ is the resonance strength of the vertical oscillation or Fourier amplitude of the driving component. For a large resonance strength, the spin can essentially completely flip over. Relative intrinsic resonance strengths for the AGS are shown in Fig. 5.

Fig. 6 shows a simulation of an interesting region of the energy ramp with two imperfection crossings, an intrinsic crossing, and a small coupling resonance. An ac dipole can be made to vertically shake the beam near the betatron tune line, so that large amplitude oscillations will enhance the spin resonance thus inducing more spin flip at the intrinsic resonance. With the ac dipole pulsed at the $\nu_{\mathrm{s}}=0+Q_{\mathrm{v}}$ intrinsic resonance, a second simulation shows a almost complete spin flip at this intrinsic resonance.

Using the CNI polarimeter in the AGS we were able to measure the polarization flips up the ramp from just above

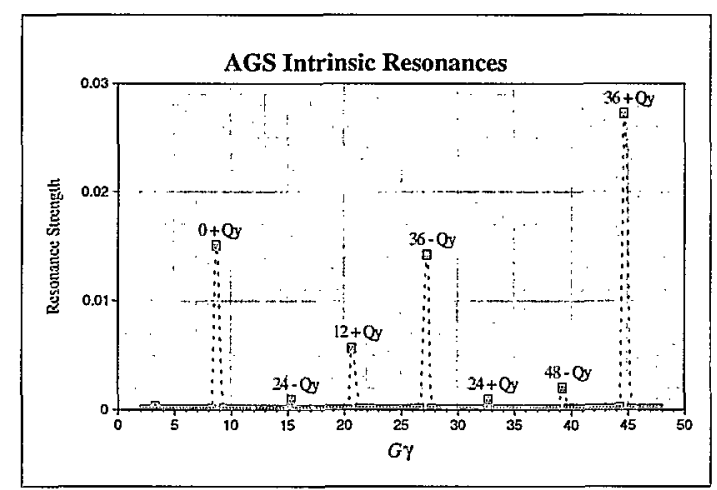

Figure 5: Calculated relative strengths of imperfection resonances in the AGS. It should be noted that there are 12 superperiods in the AGS, so the strongest intrinsic resonances occur with $N$ being a multiple of 12 and $N_{\mathrm{v}}= \pm 1$.

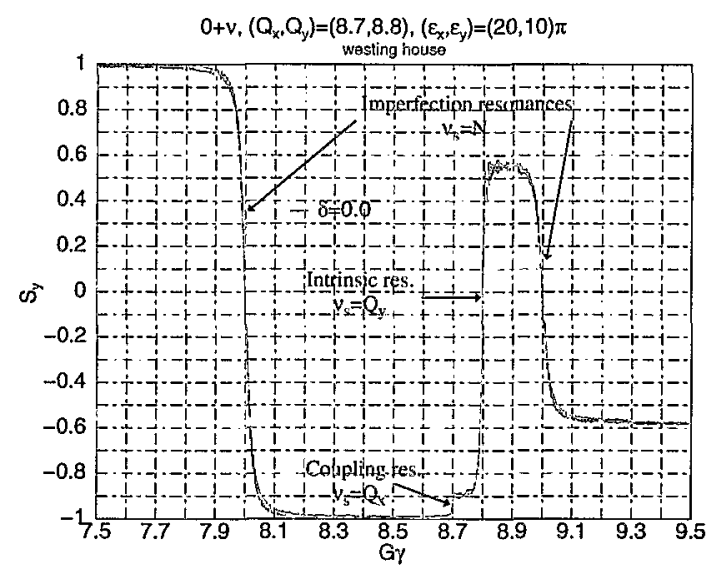

Figure 6: Simulation of resonance crossings near the first intrinsic resonance in the AGS. This shows two imperfection resonances, an intrinsic resonance and a hint of a coupling resonance.

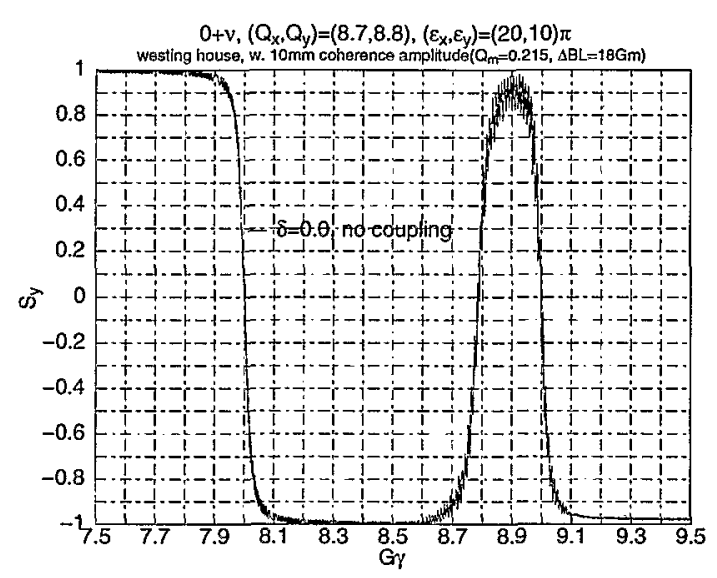

Figure 7: Simulation of the resonance crossing of Fig. 6 with an ac dipole pulse to enhance spin flipping at the intrinsic resonance. The polarization is almost completely preserved.

transition energy to the extraction energy by collecting data from many cycles over a few nights. Fig. 8 shows the raw asymmetry measurement with many flips at the integral $G \gamma$ values. We use the ac dipole to enhance spin flipping at four intrinsic resonances: $\nu_{\mathrm{s}}=0+Q_{\mathrm{v}}, 12+Q_{\mathrm{v}}, 36-Q_{\mathrm{v}}$, and $36+Q_{\mathrm{v}}$. Due to a lack of strength of the ac dipole we expect a loss of polarization at the $36+Q_{\mathrm{v}}$ with a decrease by a factor of about 0.85 at this resonance.

Fig. 9 shows a calculation of spin tracking by some large resonances in RHIC. A particle at a large amplitude oscillation sees a larger effect when crossing the resonances, and in this case does not recover the initial polarization direction. The core of the beam with smaller amplitudes shows a good retention of polarization. We have measured a decrease in polarization sometimes if the carbon filament target is placed in the tails of the beam away from the core. By severe scraping (to $1 / 6$ intensity) of the beam in the Booster 


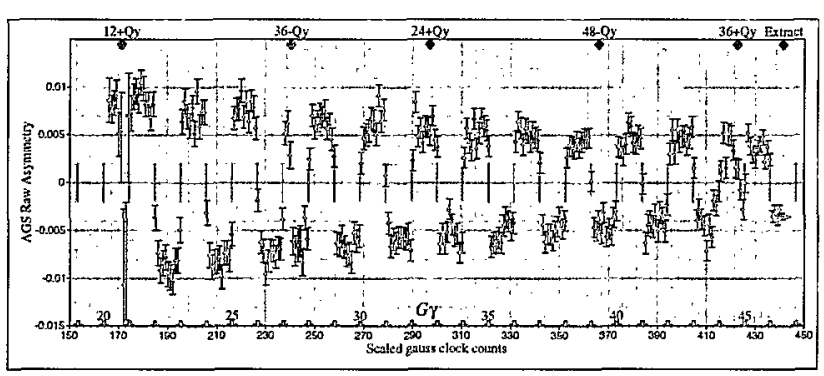

Figure 8: Measured raw asymmetry from the CNI polarimeter above transition in the AGS. Note the extra flips induced by ac dipole pulses at $12+Q_{\mathrm{v}}, 36-Q_{\mathrm{v}}$, and $36+Q_{\mathrm{v}}$. Since we do not know the energy dependence of analyzing power for this polarimeter we have plotted this as raw asymmetry rather than absolute polarization. We estimate that here the final asymmetry at extraction corresponds to about $30 \%$ polarization.

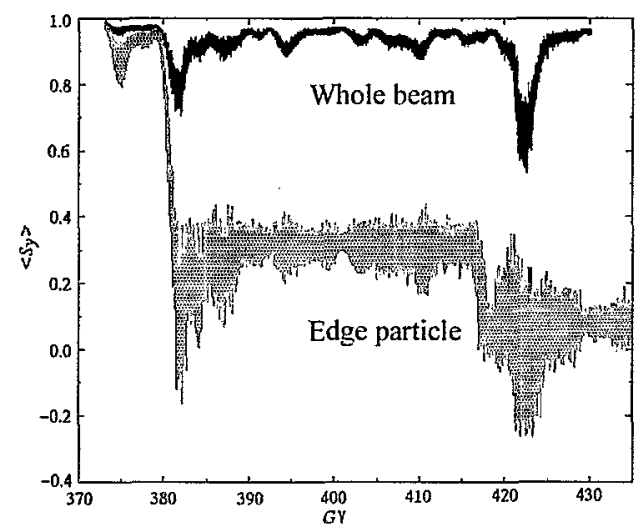

Figure 9: Calculation of spin in the region of a pair of strong resonances in RHIC. Particles with larger amplitude betatron oscillations may experience more precession away from the central stable spin direction $\vec{n}_{0}$ of the center of the beam.

in all three dimensions we can obtain a very small beam from the AGS with up to $45 \%$ or more polarization.

\section{RHIC helical Siberian snakes and rotators}

A snake is an insertion device which rotates the spin about an axis in the horizontal plane. The simplest type of snake is a solenoid which rotates the spin around the longitudinal axis. As can be seen from Eq. 1 the amount rotation from a longitudinal field decreases with energy, so a solenoid becomes less effective more higher energy machines. Another drawback of solenoids is orbital coupling. In order to maintain a spin tune of 0.5 with two snakes on opposite sides of the ring, the rotation axes of the two snakes must be kept perpendicular. If the axes were parallel, the spin tune would be zero - a bad situation for maintaining polarization.

The RHIC snakes are constructed of four helical super-
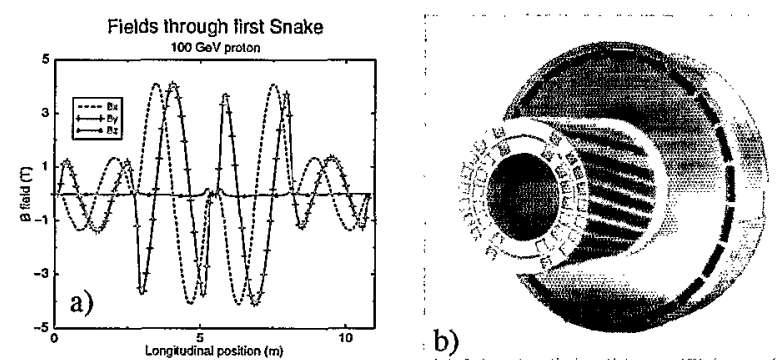

Figure 10: Superconducting helical Siberian snake. a) Magnetic fields along design orbit. The field at the end of each helix is vertical. b) Cross section of magnet showing the helical windings. The iron laminations are 14 inches in diameter.

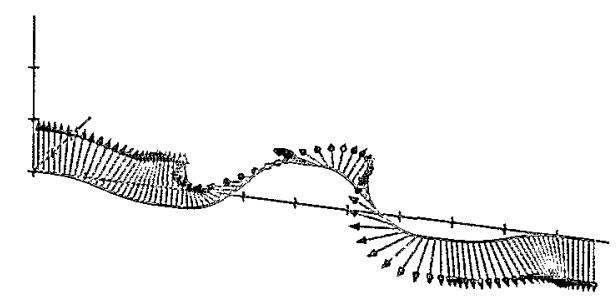

Figure 11: Trajectory and spin through a RHIC snake.

conducting dipoles with right-handed pitch and fields as indicated in Fig. 10. Fig. 11 shows the snake-like trajectory and spin rotation through a RHIC snake. In contrast with the solenoidal snake, in a helical snake spin rotation is primarily due to the transverse fields so that the rotation is essentially independent of energy. Each snake is powered by two power supplies: The outer pair of helices are connected in series with opposite polarity to one supply, and the inner pair are likewise connected in series with opposite polarity to the second supply. This orientation of fields guarantees that the snake will act as an insertion device with minimal impact on the orbit outside the snake.

There is another type of spin resonance which is driven by snakes called a snake resonance.[4] Fig. 12 shows a simulation of snake resonances for RHIC with two snakes. We have actually seen indications of the $3 / 14$ resonance showing up with decreased or zero polarization after a ramp when the horizontal tune $Q_{\mathrm{h}}$ has come close to and sometimes crossed 3/14 as shown in Fig. 13

The spin rotators for longitudinal polarization are made of four helical superconducting dipoles. In this case we alternate the handedness of the pitch rather than polarity. Moving clockwise around both rings the pattern at each rotator is: right-left-right-left. For a rotator the field at the end of each helix is horizontal. Each pair (inner and outer) of helices within a rotator is again connected in series to a different power supply. The rotation axis of this kind of rotator lies in the plane perpendicular to the beam and not necessarily in the horizontal plane as in a snake. The rotators are laid out around one of the experiments as indicated in Fig. 14

As with the snakes the amount of rotation is essentially 


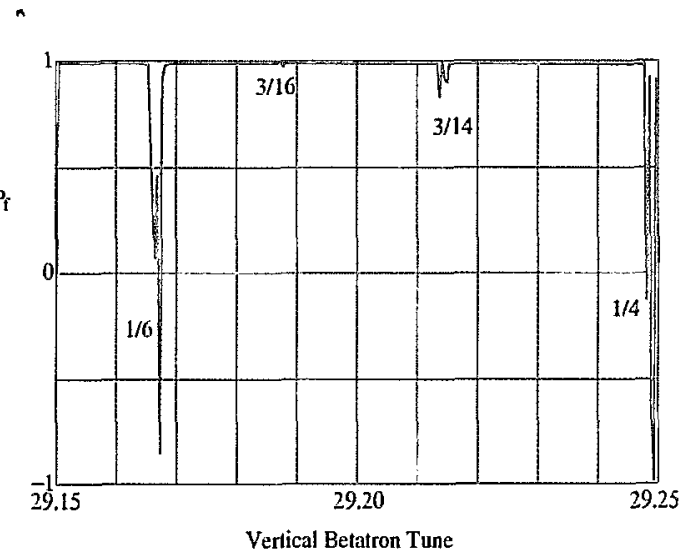

Figure 12: Simulation of hybrid snake resonances in RHIC. In RHIC we have observed depolarization with coupling when the horizontal betatron tune dips down to $3 / 14$. There is also depolarization when the vertical tune nears 0.25 .

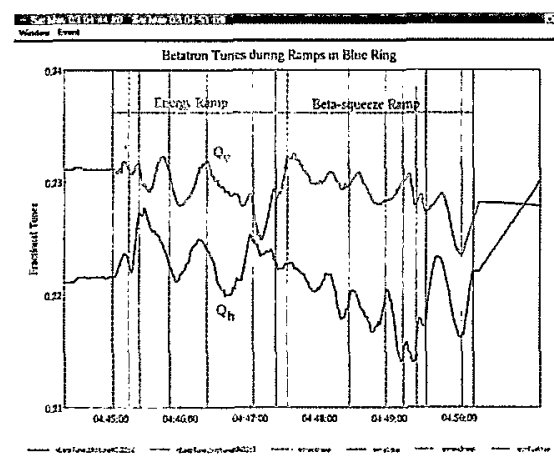

Figure 13: Measured betatron tunes in the Blue ring through the energy and and beta-squeeze ramps. The injection and energy-ramp lattice has $\beta^{*}=10 \mathrm{~m}$ at all six interaction regions in both planes. After reaching the operating energy we then squeeze the PHENIX and STAR IR's to $1 \mathrm{~m}$ and and the BRAHMS and PHOBOS IR's to $3 \mathrm{~m}$ leaving the two remaining IR's at $10 \mathrm{~m}$.

independent of energy. However due to the net horizontal bend between the rotator and interaction region there is an energy dependent precession which must be compensated to achieve a longitudinal polarization at the collision point. Fig. 15 shows how the spin precession differs at the injection and top energies.

\section{PRESENT RUN STATUS}

One of the inner helical dipoles in a Yellow ring snake failed with an open conductor. We reconfigured the snake to run with a single pair of helices by reversing the power supply connections to the outer pair of helices; with this configuration the snake can provide a rotation of $\mu=158^{\circ}$ and the required rotation axis of $\phi=-45^{\circ}$ to maintain $\nu_{\mathrm{s}}=0.5$. Rotators around PHENIX have been turned on and are providing longitudinal polarization after changing the polarity of the power supplies.[5] (With the wrong sign

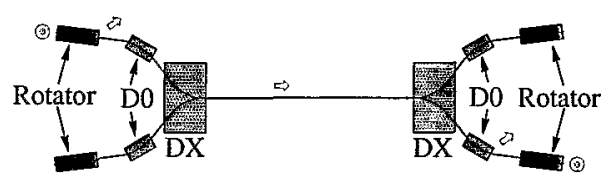

Figure 14: There are two rotators in each ring on either side of both the STAR and PHENIX detectors. Between the rotators and experiment are four dipoles (D0 and DX) to steer the beams into head-on collisions. The final-focus triplets (not indicated) are located between the rotators and and D0 magnets. For each ring the incoming rotator and outgoing rotator are parallel. There is a net bend angle of $\pm 3.675 \mathrm{mrad}$ from the rotator to the collision point.
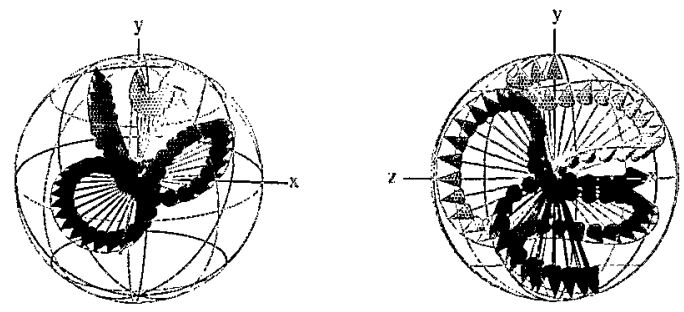

Figure 15: Spin precession through a rotator from vertical to horizontal for two energies: $24.3 \mathrm{GeV}$ (left) and $250 \mathrm{GeV}$ (right). At the lower energy the horizontal precession from the D0 and DX magnets is only about $10^{\circ}$, but at $250 \mathrm{GeV}$ it is about $100^{\circ}$.

at $100 \mathrm{GeV}$ the polarization is effectively radial. Oops.)

The injected polarization is frequently above $40 \%$ in RHIC, but we appear to be losing a quarter to a half of the polarization during the beta-squeeze ramp. Squeezing to $1 \mathrm{~m}$ at $100 \mathrm{GeV}$ causes the beam to explore the edges of the triplet aperture with strong nonlinearities and coupling. In the previous run we saw evidence[6] of the 3/14 snake resonance with coupling depolarizing the beam with $\beta^{*}=3 \mathrm{~m}$ at all six IR's. With the weak snake in the Yellow ring, the polarization at storage is not quite as good as in the Blue ring. While we have accomplished a lot in the few weeks per year dedicated to polarized protons, there is still a lot of complicated work yet to reach the full potential of the world's first polarized hadron collider.

\section{REFERENCES}

[1] I. Alekseev et al., Polarized proton collider at RHIC, NIM A 499 (2003), 392.

[2] L. H. Thomas, Phil. Mag. S. 7, 3, 1 (1927).

[3] D. P. Barber et al," "Electrons are not Protons", in EICA Workshop, Upton NY, BNL-52663 (2002).

[4] S. Y. Lee, Spin Dynamics and Snakes in Synchrotrons, World Scientific, Singapore (1997).

[5] W. W. MacKay et al., "Commissioning Spin Rotators in RHIC", These Proceedings.

[6] Vahid H. Ranjbar, Thesis "Increasing Proton Polarization in AGS and RHIC", Indiana Univ. (2002). 